\title{
Neurovascular Manifestations in Hereditary Hemorrhagic Telangiectasia: Imaging Features and Genotype- Phenotype Correlations
}

T. Krings, H. Kim, S. Power, J. Nelson, M.E. Faughnan, W.L. Young, K.G. terBrugge, and the Brain Vascular Malformation Consortium HHT Investigator Group

\begin{abstract}
BACKGROUND AND PURPOSE: Hereditary hemorrhagic telangiectasia is an autosomal dominant disease that presents in $10 \%-20 \%$ of patients with various brain vascular malformations. We aimed to report the radiologic features (phenotype) and the genotype-phenotype correlations of brain vascular malformations in hereditary hemorrhagic telangiectasia.
\end{abstract}

MATERIALS AND METHODS: Demographic, clinical, genotypic, and imaging information of 75 patients with hereditary hemorrhagic telangiectasia with brain arteriovenous malformations enrolled in the Brain Vascular Malformation Consortium from 2010 to 2012 were reviewed.

RESULTS: Nonshunting, small, superficially located conglomerates of enhancing vessels without enlarged feeding arteries or draining veins called "capillary vascular malformations" were the most commonly observed lesion (46 of 75 patients; 61\%), followed by shunting "nidus-type" brain AVMs that were typically located superficially with a low Spetzler-Martin Grade and a small size (32 of 75 patients; $43 \%$ ). Direct high-flow fistulous arteriovenous shunts were present in 9 patients (12\%). Other types of vascular malformations (dural AVF and developmental venous anomalies) were present in 1 patient each. Multiplicity of vascular malformations was seen in 33 cases (44\%). No statistically significant correlation was observed between hereditary hemorrhagic telangiectasia gene mutation and lesion type or lesion multiplicity.

CONCLUSIONS: Depending on their imaging features, brain vascular malformations in hereditary hemorrhagic telangiectasia can be subdivided into brain AVF, nidus-type AVM, and capillary vascular malformations, with the latter being the most common phenotype in hereditary hemorrhagic telangiectasia. No genotype-phenotype correlation was observed among patients with this condition.

ABBREVIATIONS: $\mathrm{CVM}=$ capillary vascular malformation; $\mathrm{HHT}=$ hereditary hemorrhagic telangiectasia

$\mathrm{H}_{\mathrm{b}=\mathrm{s}}$ ereditary hemorrhagic telangiectasia (HHT) or Rendu-Weber-Osler disease is a familial disorder that occurs with a prevalence of approximately $1 / 10,000 .{ }^{1-3}$ The diagnostic certainty of HHT is determined by the number of characteristic clinical findings present in an individual patient. These clinical findings

Received August 26, 2014; accepted after revision October 8.

From the Division of Neuroradiology (T.K., S.P., K.G.t.B.), Department of Medical Imaging, and Division of Neurosurgery (T.K.), Department of Surgery, Toronto Western Hospital, University of Toronto, Toronto, Ontario, Canada; Department of Anesthesia and Perioperative Care (H.K., J.N., W.L.Y.), Center for Cerebrovascular Research, and Department of Epidemiology and Biostatistics (H.K.), University of California San Francisco, San Francisco, California; Division of Respirology (M.E.F.), Department of Medicine and Li Ka Shing Knowledge Institute, St. Michael's Hospital, Toronto, Ontario, Canada; and Division of Respirology (M.E.F.), Department of Medicine, University of Toronto, Toronto, Ontario, Canada.

The Brain Vascular Malformation Consortium (U54NS065705) is a part of the National Institutes of Health Rare Diseases Clinical Research Network, supported through collaboration between the National Institutes of Health Office of Rare Diseases Research at the National Center for Advancing Translational Sciences and the National Institute of Neurological Disorders and Stroke. Other financial support to M. Faughnan is from the Nelson Arthur Hyland Foundation and the Li Ka Shing Knowledge Institute.

The content of this article is solely the responsibility of the authors and does not necessarily represent the official views of the National Institutes of Health. are the following: 1) nosebleeds, 2) mucocutaneous telangiectasias (of the lips, oral cavity, nose, or fingers), 3) AVMs (of the lungs, the gastrointestinal system, or the CNS), and 4) an affected first-degree relative. In "definite" HHT, 3 of these clinical criteria are present, while "suspected" or "unlikely" HHT diagnoses consist of 2 or 1 item present, respectively. It is estimated that $10 \%-$ $20 \%$ of patients with HHT have brain vascular malformations ${ }^{4}$ with additional neurovascular complications from pulmonary AVMs (stroke and cerebral abscess). ${ }^{5}$

HHT is inherited as an autosomal dominant disorder, caused by mutation in 1 of 3 genes identified to date: endoglin (HHT1) on chromosome 9q34; activin receptor-like kinase 1 or ALK1 (HHT2) on chromosome 12q13; and SMAD4 on chromosome 18q21 (juvenile polyposis, HHT overlap syndrome) ${ }^{6-10}$ The associated

Please address correspondence to Timo Krings, MD, PhD, Division of Neuroradiology, Department of Medical Imaging, Toronto Western Hospital, 399 Bathurst St, 3 McL, Toronto, ON, M5T 2S8, Canada; e-mail: Timo.Krings@uhn.ca

-- Indicates open access to non-subscribers at www.ajnr.org

三 Indicates article with supplemental on-line table.

http://dx.doi.org/10.3174/ajnr.A4210 
gene products are expressed in endothelial cells and are part of the transforming growth factor- $\beta$ signaling pathway, thus involved in angiogenesis and vascular remodeling. Endothelial cells lacking functioning endoglin or ALK1 form abnormal vessels and abnormal connections between vessels. ${ }^{6}$ Early HHT genotype-phenotype correlation studies demonstrated an association between endoglin mutation and brain AVMs, ${ }^{1-13}$ though more recent studies have demonstrated that brain AVMs can be present with all HHT genotypes. ${ }^{14,15}$ No studies to date have addressed HHT brain AVM phenotypes and their correlation with genotype, to our knowledge.

To date, in the 2 largest single-center series of brain AVMs in HHT with 52 and 14 patients, respectively, 3 different distinct phenotypes of brain vascular malformations were described independently: 1) high-flow "single-hole" pial fistulas, 2) "classic" nidus-type brain AVMs, and 3) "micro-AVM" or "capillary vascular malformations," defined as small lesions without clear evidence of a shunt. ${ }^{5,16}$

The aim of the present series was 2-fold: 1) to identify the different radiologic and, in particular angiographic features of brain vascular malformations in HHT, and to subclassify them into different types, and 2) to determine whether there is a genotype-phenotype correlation between the HHT gene mutation and the type of vascular malformation.

\section{MATERIALS AND METHODS Study Population}

We analyzed data from patients recruited to the HHT Project of the Brain Vascular Malformation Consortium. Patients with HHT with a confirmed clinical HHT diagnosis by the Curaçao criteria $^{17}$ or a confirmed genetic diagnosis were enrolled as part of the Brain Vascular Malformation Consortium HHT Project as previously described (http://rarediseasesnetwork.epi.usf.edu/ BVMC) ${ }^{18}$ All patients provided written, informed consent, including consent for genetic studies. The study protocol was approved by each institutional review board. Data collected included age, sex, family relationships, genetic testing results (mutated gene: ACVRL1, ENG, SMAD4, and unknown), clinical presentation, and symptoms. Patients were screened for AVM and other HHT clinical features according to standard clinical practice and international HHT guidelines. ${ }^{19}$ The Brain Vascular Malformation Consortium HHT cohort targets patients positive for brain AVM but enrolls all HHT cases in a 1:4 ratio, respectively. Cohort characteristics are otherwise similar to those in other cohorts. ${ }^{12,14}$

Between 2010 and 2012, comprehensive data were available for 384 recruited patients with HHT, 125 of whom had brain AVMs, with sufficient diagnostic imaging available for review in 75 of these patients with brain AVMs. All patients provided written, informed consent, and the study protocol was approved by each institutional review board.

\section{Data Collection}

Epidemiologic data collected included age at enrollment and diagnosis, sex, and HHT mutation type. Radiologic data included MR imaging (with contrast-enhanced sequences in at least 2 planes on $1.5 \mathrm{~T}$ or $3 \mathrm{~T}$ scanners) and angiography with multiphase biplanar selective 4-vessel angiography. The angiographic parameters that were identified for every patient who was identified with a brain vascular malformation are listed in the On-line Table. In addition to location, size in the largest dimension, and eloquence (as determined by the Spetzler-Martin classification), specific information about feeding arteries (such as the degree of dilation, number, type [choroidal versus pial], associated aneurysms, and so forth), the nidus (glomus versus fistula, diffuse versus sharp, perinidal angiogenesis, and so forth), and the draining veins (associated pouches, degree of dilation, associated stenosis, number, deep versus superficial, and so forth) was noted. Images were reviewed by 2 of the authors (T.K. and S.P.) in consensus; in cases of disagreement, a third neuroradiologist (K.G.t.B.) reviewed the case.

On the basis of previous work and our observations in the present study, we were able to subclassify 3 types of vascular malformations: the pial arteriovenous fistula (defined by the absence of an intervening nidus between the feeding artery and draining vein in the presence of a shunt), the nidus-type AVM (defined by the presence of a shunt with early filling of a vein through a dilated network of abnormal vessels), and a "capillary malformation," in which no shunt was visible on angiography and no dilated feeding arteries or veins were identified; just a blush of abnormal vessels was seen in the capillary phase. Vascular malformations that did not fit into any of these categories (developmental venous anomalies, dural arteriovenous fistulas, pontine capillary telangiectasias, cavernomas, and so forth) were specifically sought and, if present, were recorded.

\section{Statistical Tests}

We tested for differences of age at diagnosis, sex, and HHT gene mutation (ALK1 versus endoglin) between patients with AVFs and those without AVFs. Similarly, we tested for differences in characteristics between patients with capillary vascular malformations (CVMs) and those without and for patients with AVMs and those without. Additionally, we checked whether age, sex, or HHT gene mutation was associated with lesion multiplicity ( $\geq 2$ lesions of any type). Comparisons involving age were tested with 2 -sample $t$ tests, allowing for unequal variances, while comparisons involving sex or mutation differences were tested by using Fisher exact tests. We considered $P$ values $<.05$ to be statistically significant. All analyses were performed using STATA/SE 13.1 (StataCorp, College Station, Texas).

\section{RESULTS}

A summary of demographic and clinical features of the 75 HHT subjects with brain AVMs is presented in Table 1. The average age at enrollment for these subjects was 37 years, and the average age at brain malformation diagnosis was 30 years. Women were slightly more prevalent in the sample (55\%). HHT gene mutation status was available for $60 \%$ of participants, and among those, $60 \%$ had an endoglin mutation.

On the basis of the criteria described in the "Materials and Methods" section, we identified 3 different types of vascular malformations: capillary vascular malformations, AVMs, and AVFs. Table 2 details the distribution of these lesions across subjects. Capillary vascular malformations were the most commonly 
Table 1: Overview of 75 subjects with HHT and brain vascular malformations

\begin{tabular}{lc}
\hline \multicolumn{1}{c}{ Characteristic } & Summary $^{\text {a }}$ \\
\hline Demographics & \\
Female sex & $41 / 75(55 \%)$ \\
Age at enrollment $(y r)(n=75)$ & $36.6 \pm 19.9$ \\
Age at brain malformation diagnosis $(y r)(n=68)$ & $30.1 \pm 19.7$ \\
HHT-related symptoms & \\
Epistaxis & $66 / 73(90 \%)$ \\
Anemia & $20 / 72(28 \%)$ \\
GI bleeding & $5 / 67(7 \%)$ \\
Pulmonary AVM & $45 / 69(65 \%)$ \\
Liver VM & $4 / 66(6 \%)$ \\
HHT-causing mutation & \\
ALK1 & $13 / 45(29 \%)$ \\
Endoglin & $27 / 45(60 \%)$ \\
SMAD4 & $1 / 45(2 \%)$ \\
All test findings negative & $4 / 45(9 \%)$ \\
\hline
\end{tabular}

Note:-GI indicates gastrointestinal; VM, vascular malformations.

a Summary is the No. of observations with specified value over the total No. (and the percentage) or the mean $\pm \mathrm{SD}$.

Table 2: Brain vascular malformation phenotypes of 75 subjects with HHT

\begin{tabular}{|c|c|c|c|}
\hline & \multicolumn{3}{|c|}{ Capillary Vascular Malformation } \\
\hline & 0 & 1 & $2+$ \\
\hline $0 \mathrm{AVMs} / \mathrm{AVFs}$ & $1(1 \%)^{\mathrm{a}}$ & $17(23 \%)$ & $18(24 \%)^{b}$ \\
\hline $1 \mathrm{AVM}$ & $20(27 \%)$ & $5(7 \%)$ & $3(4 \%)$ \\
\hline 2 AVMs & $1(1 \%)$ & $0(0 \%)$ & $1(1 \%)$ \\
\hline $1 \mathrm{AVF}$ & $4(5 \%)$ & $1(1 \%)$ & $0(0 \%)$ \\
\hline $2 \mathrm{AVFs}$ & $1(1 \%)$ & $1(1 \%)$ & $0(0 \%)$ \\
\hline $1 \mathrm{AVM}$ and $1 \mathrm{AVF}$ & $2(3 \%)$ & $0(0 \%)$ & $0(0 \%)$ \\
\hline
\end{tabular}

a This individual had 1 dural arteriovenous fistula only.

${ }^{\mathrm{b}}$ One individual in this group had a developmental venous anomaly.

Table 3: Characteristics of 80 capillary vascular malformations

\begin{tabular}{lc}
\hline \multicolumn{1}{c}{ Characteristic } & Summary $^{\mathrm{a}}$ \\
\hline Size of maximal dimension $(\mathrm{mm})(n=80)$ & $4.8 \pm 2.1$ \\
Lobe & \\
Frontal & $30 / 80(38 \%)$ \\
Parietal & $16 / 80(20 \%)$ \\
Occipital & $14 / 80(18 \%)$ \\
Temporal & $10 / 80(13 \%)$ \\
Cerebellum & $7 / 80(9 \%)$ \\
Brain stem & $2 / 80(3 \%)$ \\
Thalamus/basal ganglia & $1 / 80(1 \%)$ \\
Location & \\
Deep & $3 / 80(4 \%)$ \\
Subcortical & $14 / 80(18 \%)$ \\
Superficial & $63 / 80(79 \%)$ \\
MRI visible & $66 / 71(93 \%)$ \\
\hline
\end{tabular}

a Summary is the No. of observations with specified value over the total No. (and the percentage) or the mean \pm SD.

observed lesion, with $61 \%$ of subjects having $\geq 1$ CVM. Fortythree percent of subjects had $\geq 1 \mathrm{AVM}$, and $12 \%$ had $\geq 1 \mathrm{AVF}$. One subject had a dural arteriovenous fistula, and 1 subject had a developmental venous anomaly. Multiplicity of lesions was common: Thirty-three $(44 \%)$ subjects had $\geq 2$ brain malformations.

Tables 3-5 present the typical imaging features of the different vascular malformations: CVMs were identified as lesions that had no dilated feeder and no dilated draining veins (Figs 1-3). Eighty of these lesions were identified in 46 patients. All of these lesions were $<1 \mathrm{~cm}$; most were located in the superficial supratentorial
Table 4: Characteristics of 34 AVMs

\begin{tabular}{|c|c|}
\hline Characteristic & Summary $^{a}$ \\
\hline Size of maximal dimension $(\mathrm{mm})(n=34)$ & $18.3 \pm 5.3$ \\
\hline Eloquent & $13 / 34(38 \%)$ \\
\hline Deep venous drainage & $5 / 31(16 \%)$ \\
\hline \multicolumn{2}{|l|}{ Spetzler-Martin score } \\
\hline 1 & $16 / 31(52 \%)$ \\
\hline 2 & $12 / 31(39 \%)$ \\
\hline 3 & 2/31 (6\%) \\
\hline 4 & $1 / 31(3 \%)$ \\
\hline 5 & $0 / 31(0 \%)$ \\
\hline \multicolumn{2}{|l|}{ Lobe } \\
\hline Frontal & $14 / 34(41 \%)$ \\
\hline Parietal & $5 / 34(15 \%)$ \\
\hline Occipital & $4 / 34(12 \%)$ \\
\hline Temporal & $5 / 34(15 \%)$ \\
\hline Cerebellum & $2 / 34(6 \%)$ \\
\hline Brain stem & $2 / 34(6 \%)$ \\
\hline Thalamus/basal ganglia & $2 / 34(6 \%)$ \\
\hline \multicolumn{2}{|l|}{ Location } \\
\hline Deep & $4 / 34(12 \%)$ \\
\hline Subcortical & $5 / 34(15 \%)$ \\
\hline Superficial & $25 / 34(74 \%)$ \\
\hline Nidus en passage (vs terminal) & $2 / 20(10 \%)$ \\
\hline Diffuse nidus (vs sharp) & $6 / 34(18 \%)$ \\
\hline Pial-to-pial collateralization & $5 / 31(16 \%)$ \\
\hline Moyamoya-type changes & $0 / 33(0 \%)$ \\
\hline Stenoses on arteries & 0/31 (0\%) \\
\hline Type, pial (vs choroidal) & $33 / 34(97 \%)$ \\
\hline Associated aneurysms & $0 / 30(0 \%)$ \\
\hline Multiple draining veins & $3 / 29(10 \%)$ \\
\hline Venous ectasia & $8 / 31(26 \%)$ \\
\hline Venous reflux & 1/29 (3\%) \\
\hline Pseudophlebitic pattern & $0 / 29(0 \%)$ \\
\hline
\end{tabular}

a Summary is the No. of observations with specified value over the total No. (and the percentage) or the mean \pm SD.

\section{Table 5: Characteristics of 11 AVFs}

\begin{tabular}{lc}
\hline \multicolumn{1}{c}{ Characteristic } & Summary ${ }^{\mathbf{a}}$ \\
\hline Single hole with pouch & $10 / 11(91 \%)$ \\
Lobe & \\
Frontal & $4 / 11(36 \%)$ \\
Parietal & $0 / 11(0 \%)$ \\
Occipital & $3 / 11(27 \%)$ \\
Temporal & $3 / 11(27 \%)$ \\
Cerebellum & $0 / 11(0 \%)$ \\
Brain stem & $0 / 11(0 \%)$ \\
Thalamus/basal ganglia & $1 / 11(9 \%)$ \\
Location & \\
Deep & $1 / 11(9 \%)$ \\
Subcortical & $0 / 11(0 \%)$ \\
Superficial & $10 / 11(91 \%)$ \\
Pial-to-pial collateralization & $3 / 11(27 \%)$ \\
Moyamoya-type changes & $1 / 11(9 \%)$ \\
Stenoses on arteries & $1 / 11(9 \%)$ \\
Type, pial (vs choroidal) & $2 / 11(18 \%)$ \\
Associated aneurysms & $2 / 11(18 \%)$ \\
Multiple draining veins & $5 / 11(45 \%)$ \\
Venous ectasia & $11 / 11(100 \%)$ \\
Venous reflux & $0 / 11(0 \%)$ \\
Pseudophlebitic pattern & $3 / 11(27 \%)$ \\
\hline
\end{tabular}

a Summary is the No. of observations with specified value over the total No. (and the percentage).

brain. On MR imaging, they appeared as small foci of "fluffy" enhancement without abnormal vascular dilation. There was no edema surrounding these vascular malformations. Thirty-four 

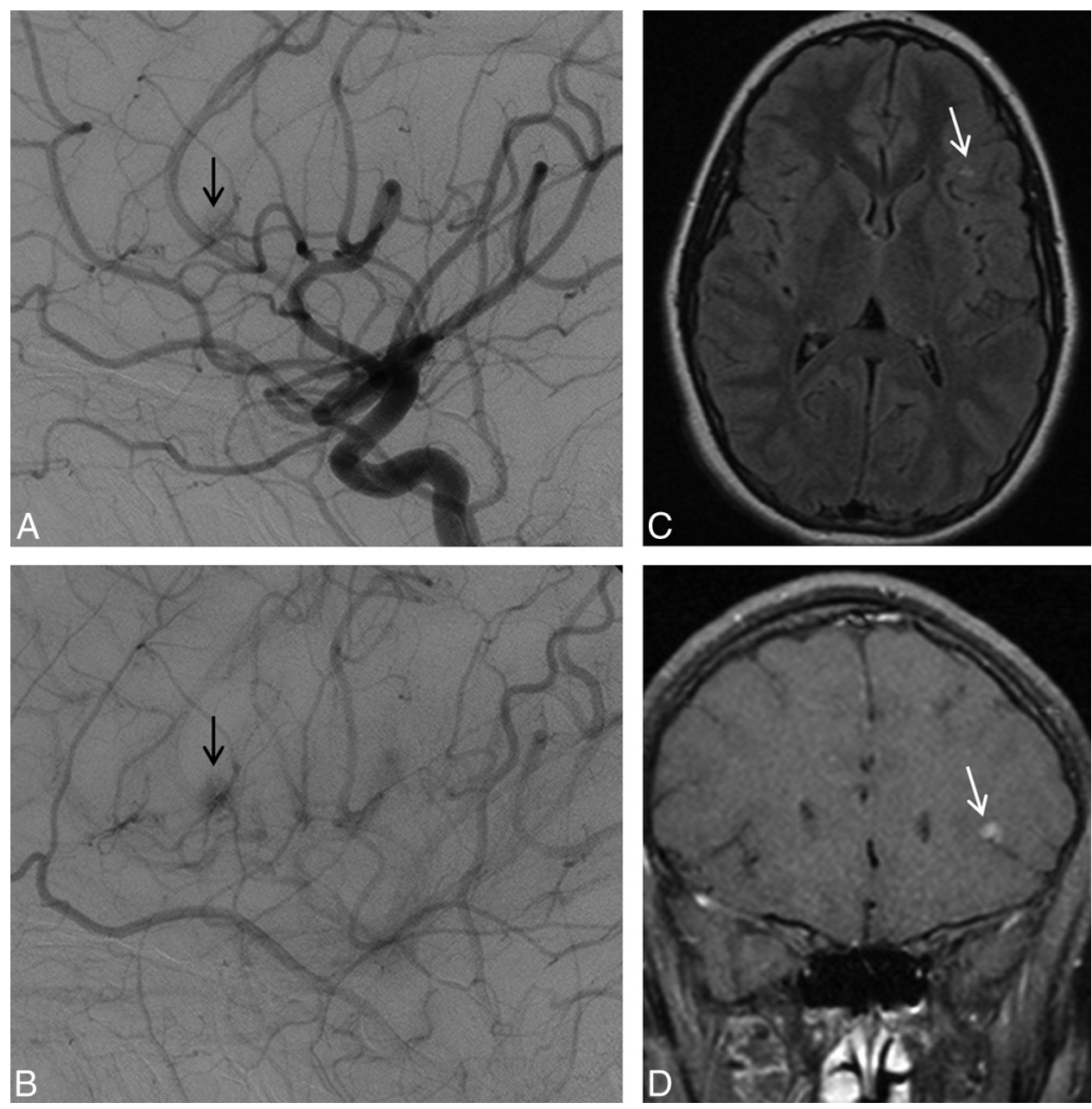

FIG 1. Left ICA angiogram, lateral view, depicts typical features of a capillary vascular malformation in the left frontal opercular region, with a subcentimeter vascular blush identified in the arterial $(A)$ persisting into early venous phase ( $B$, arrow), without evidence of arteriovenous shunting. On MR imaging, this appears as a focal region of hyperintensity on axial T2 FLAIR imaging ( $C$, arrow), with fluffy enhancement on coronal gadolinium-enhanced T1-weighted imaging $(D$, arrow). No dilated arterial feeder or dilated draining vein is seen.

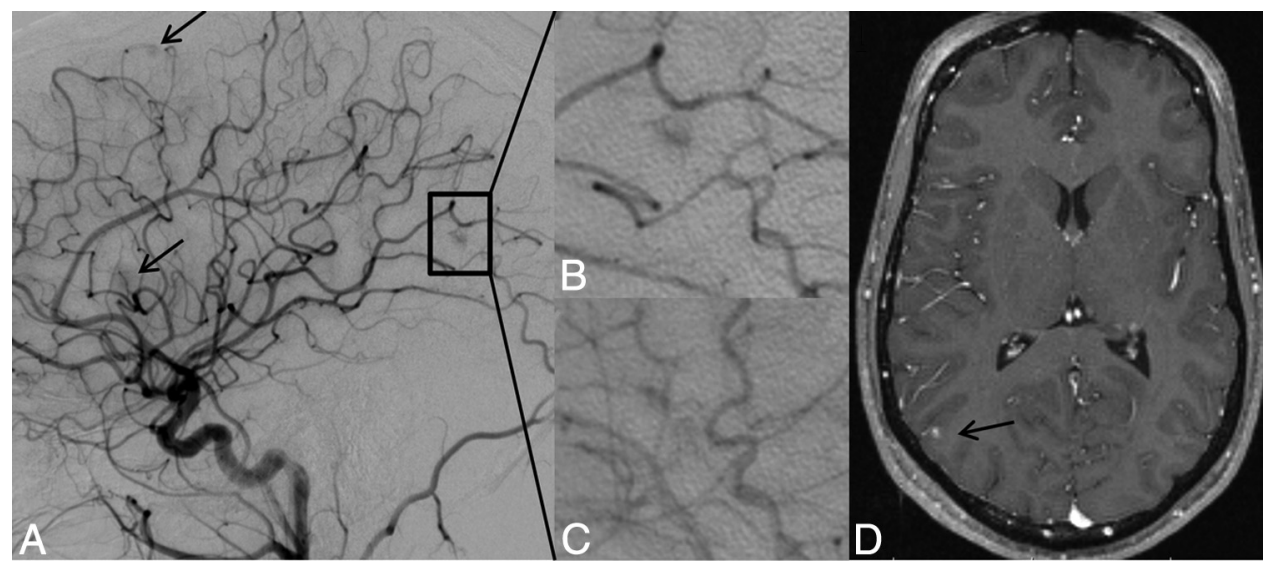

FIG 2. Lateral projection of right ICA angiogram $(A-C)$ and axial TT-weighted gadolinium-enhanced $M R$ imaging $(D)$ demonstrate the typical imaging features of a capillary vascular malformation in a 17-year-old male patient with HHT. A focal subcentimeter blush (arrows) is identified on the arterial phase of a right carotid angiogram, in the frontal, temporal, and parietal regions, in keeping with multiple capillary telangiectasia. Magnified view of the right parietal lesion shows typical angiographic features, with vascular blush identified in the arterial $(B)$ persisting into early venous phase $(C)$; no arteriovenous shunting, dilated arterial feeder, or dilated draining vein are seen. MR imaging appearance of the right parietal lesion demonstrates the typical features of fluffy contrast enhancement in a superficial cortical/subcortical location, without abnormal vascular dilation (D, arrow).

AVMs were present in 32 patients (Figs 4 and 5). As the major differentiating factor from CVMs, the AVMs had enlarged feeding arteries and draining veins and a shunt was visible on angiogra- phy. In all, an intervening nidus of abnormal vessels was identified. Lesions were almost always $<3 \mathrm{~cm}$ and rarely (in 2 cases) $<1$ $\mathrm{cm}$. They had a compact, well-defined nidus and were mainly 
located supratentorially and superficially. Given the superficial location and the small size, their Spetzler-Martin grade was typically $1-2$, with the higher grades mainly driven by their potential location in eloquent cortex. Feeding arteries were almost always pial vessels (we encountered only 1 choroidal AVM in this series) and were classically fed by terminal-type feeders. Associated aneurysms or other signs for high flow (pial-to-pial collaterals, venous ectasias, and so forth) were uncommon. On the venous side, venous reflux or corkscrew-like dilated veins as signs of long-standing venous hypertension were typically absent. Eleven AVFs were present in 9 patients; these were typically high-flow single-hole macrofistulas that were located in the supratentorial superficial brain with significant venous ectasia and signs of high flow with consecutive perilesional hypoxemia (Figs 6 and 7).

We found that patients with AVFs tended to be diagnosed at younger ages than those without $\operatorname{AVFs}(P=.028)$; the average age at diagnosis for patients with AVFs was 15 years compared with an average age of 32 years for patients without AVFs. There was no significant association between the presence of AVFs and sex $(P=$ $.723)$ or HHT gene mutation $(P=1.000)$. No significant difference was observed with age, sex, or HHT gene mutation in those patients with capillary vascular malformations versus no CVMs or in patients with AVMs versus those without AVMs. Lesion multiplicity was also not significantly associated with age $(P=$ .736), sex $(P=.484)$, or HHT gene mutation $(P=1.000)$.
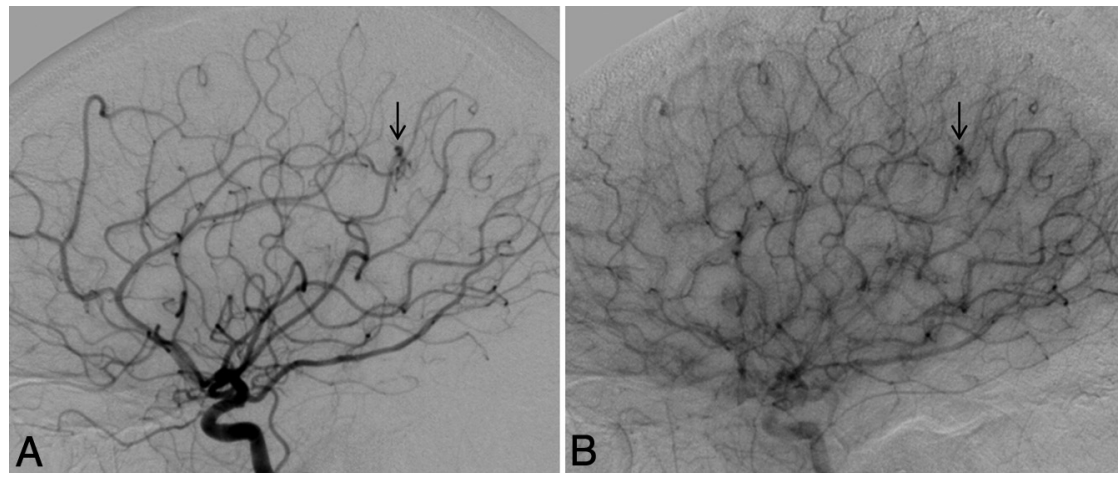

FIG 3. Lateral view of a right ICA angiogram in a 43-year-old male patient with HHT demonstrates a capillary vascular malformation in the right anterior parietal region. A subcentimeter blush is seen in the arterial phase $(A$, arrow), which persists in the late arterial phase $(B)$ without evidence of arteriovenous shunting.

\section{DISCUSSION}

We report herein the largest series and most comprehensive description of brain AVM phenotype among patients with HHT.

On the basis of radiologic and angiographic information, we were able to subclassify 3 different brain vascular malformations in patients with HHT that had some common and distinctive imaging features: The common finding was that brain vascular malformations in HHT tended to be located in supratentorial and superficial compartments. Deep or infratentorial locations were

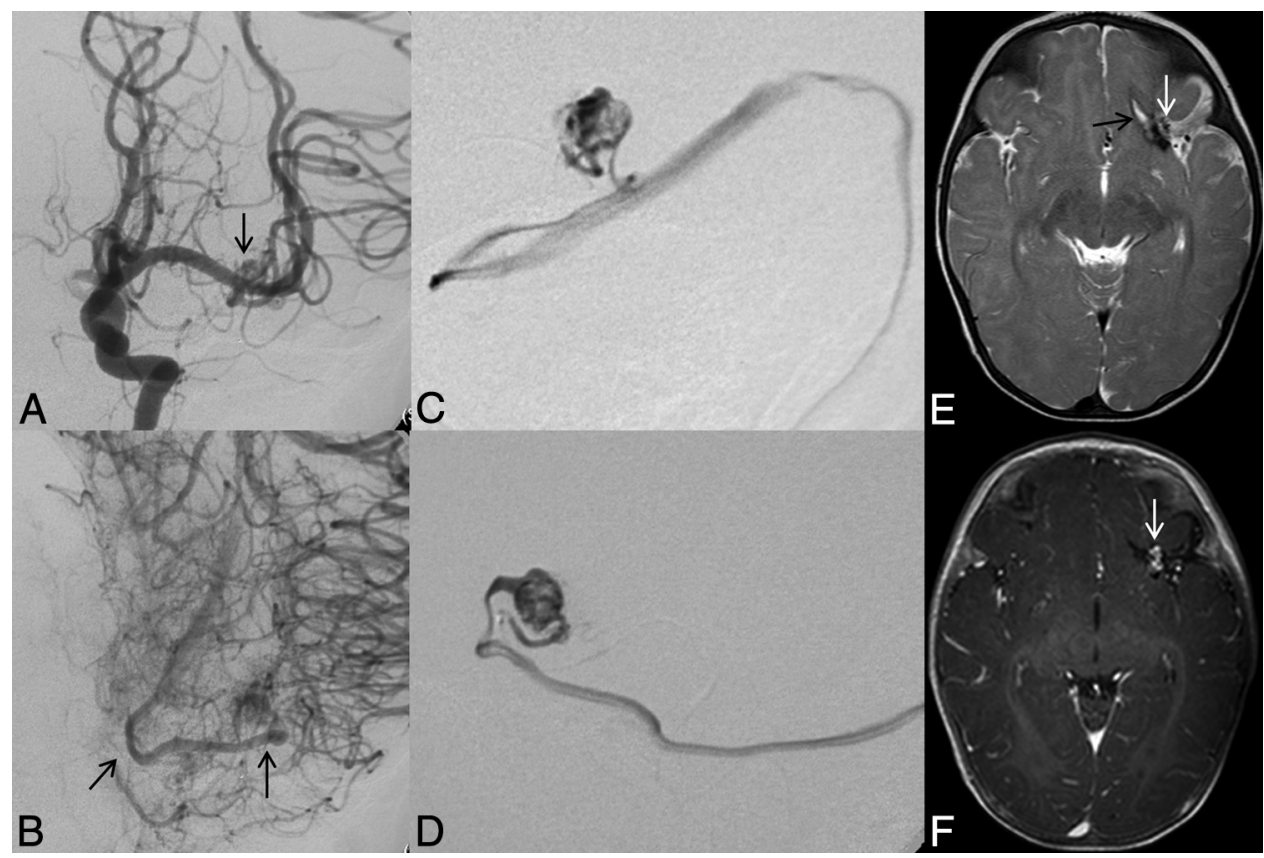

FIG 4. Left ICA catheter angiogram ( $A$ and $B)$, microcatheter injection ( $C$ and $D)$, axial T2-weighted $(E)$, and contrast-enhanced T1-weighted MR imaging $(F)$ demonstrate imaging findings of a typical arteriovenous malformation. Anteroposterior projection of a left ICA injection demonstrates filling of the AVM nidus through an enlarged anterior temporal branch of the left MCA in the early arterial phase (A, arrow). There is arteriovenous shunting with early venous drainage through an enlarged left inferior temporal vein $(B$, arrows). Microcatheter injection in frontal $(C)$ and lateral $(D)$ projections demonstrates a typical glomerular well-defined compact nidus supplied by a single terminal arterial feeder with shunting into a dilated vein. MR imaging shows the superficial location of the AVM, with vascular flow voids seen on T2-weighted imaging (E, arrow) and enhancement on postgadolinium Tl-weighted imaging ( $F$, arrow). Evidence of previous hemorrhage related to AVM rupture, with a slit-like hematoma cavity in the left inferior frontal lobe, and surrounding hemosiderin staining ( $E$, black arrow). 

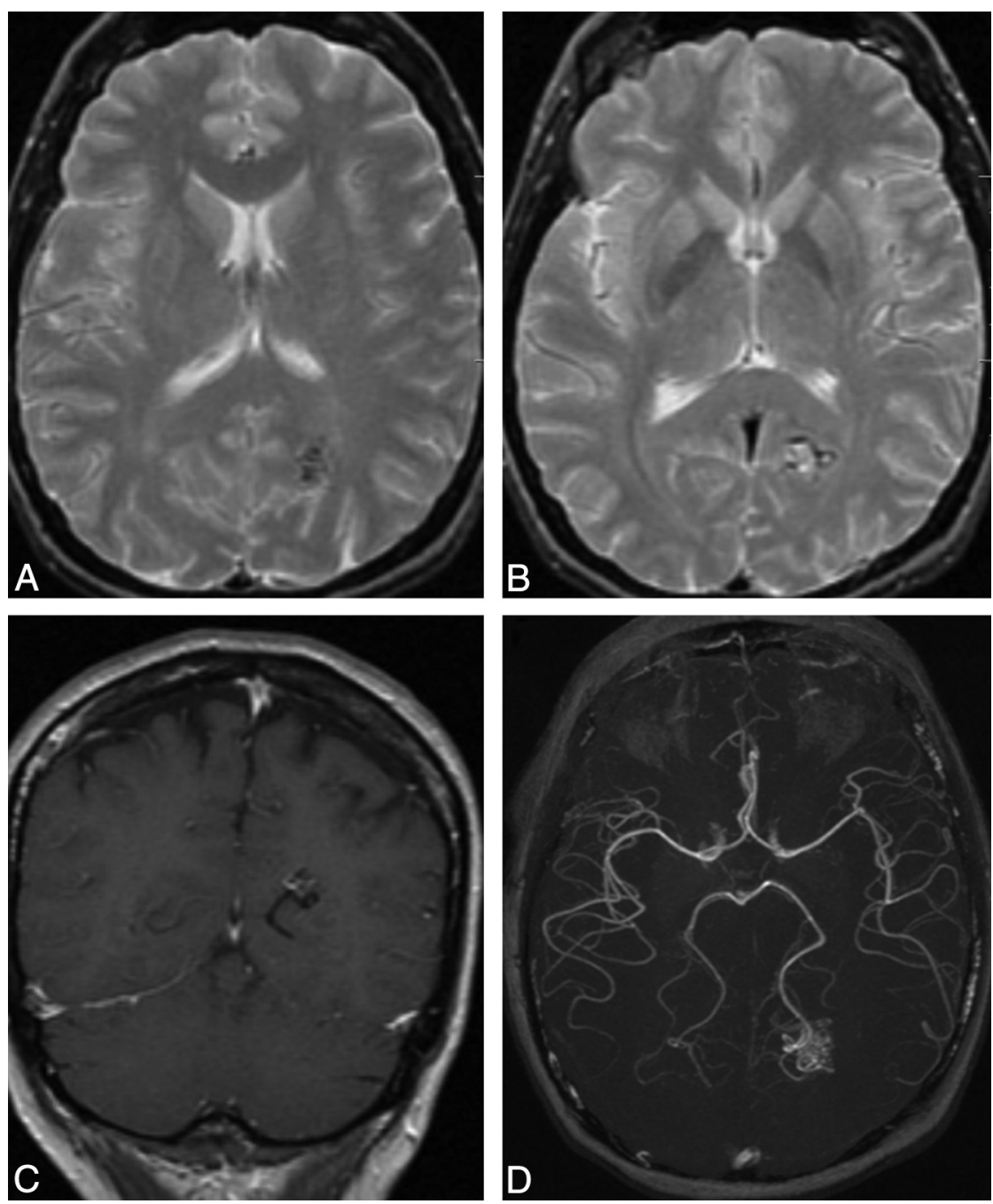

their Spetzler-Martin grade tended to be low. Again, this is in keeping with the findings in previous literature. The socalled capillary vascular malformations did not present with dilated vessels (neither on the feeding artery nor the draining venous side) and demonstrated a focal area of fluffy enhancement on MR imaging.

In this article, we introduce the term "capillary vascular malformation" for those vascular malformations that do not exhibit enlarged arteries or veins on MR imaging and in which no true shunt is present on angiography. These vascular malformations have been previously coined "micro-AVMs," which may lead to the assumption that they behave in a manner similar to true brain AVMs. However, because these vascular lesions differ from "AVMs" in their angioarchitecture, one may hypothesize that they also behave differently in their clinical presentation, an assumption that is founded on the combined experience of the Toronto $^{5}$ and the Bicêtre ${ }^{16}$ articles, in which no hemorrhages were encountered for these types of malformations. While in AVMs an arteriovenous shunt is present due to lack of a true capillary bed, the term "capillary malformation" tries to highlight the absence of a shunt and the presence of a capillary bed that is abnormally dilated (and thus visible as a blush within the capillary phase of the angiography and faint enhancement on MR imaging).

We found, similar to findings in previous series, ${ }^{20}$ no significant sex predi-

atypical. This finding corresponds well with the literature: In the Bicêtre series, 61 of 68 brain vascular malformations (90\%) were located superficially and 45 of 68 lesions (66\%) were supratentorial. ${ }^{16}$ Lesion identification by MR imaging was always possible for the AVMs and AVFs; however, a few capillary vascular malformations could not be detected by MR imaging alone. Distinguishing features among the 3 subtypes were the presence or absence of a shunt and, if a shunt was present, whether the abnormal arteriovenous communication was direct (ie, fistulous) or through a network (or nidus).

Pial AVFs typically had a high shunt volume and therefore often led to massive enlargement of the feeding arteries, significant venous ectasia, and, due to locoregional hypoxemia, secondary changes such as perinidal angiogenesis. We presume that the relatively higher proportion of direct fistulas in the Bicêtre series was related to a referral bias because these lesions tend to be more commonly found in children. ${ }^{16}$ Brain AVMs in our series tended to be small (typically $<3 \mathrm{~cm}$ in their largest diameter and, in 2 cases, $<1 \mathrm{~cm}$ ), and because their location was typically superficial, lection, a rather young overall age at presentation, and a high propensity for multiplicity of brain lesions if present. Our proportion of multiplicity ( 33 in 75 patients $=44 \%$ ) was very similar to numbers reported previously ( 23 of 56 patients $=39 \%$ in the series of Bharatha et $\mathrm{al}^{20} ; 16$ of $44=36 \%$ in the Bicêtre series, when taking into account only the cerebral manifestations ${ }^{16}$ ). Given a rate of approximately $1 \%$ multiplicity of brain vascular malformations in the non-HHT population, our data support the previously described high predictive value of AVM multiplicity for the diagnosis of HHT.

Our observations are in keeping with previous reports on the neurovascular manifestations in HHT, but we think that proof of findings of these single-center studies from a multicenter consortium is still necessary and worthwhile reporting. To date, most studies on brain vascular malformations in HHT do not subclassify the different types of vascular malformations. However, it is quite conceivable that the various types will have different natural histories and therefore warrant different treatments. In neither of the 2 larger 


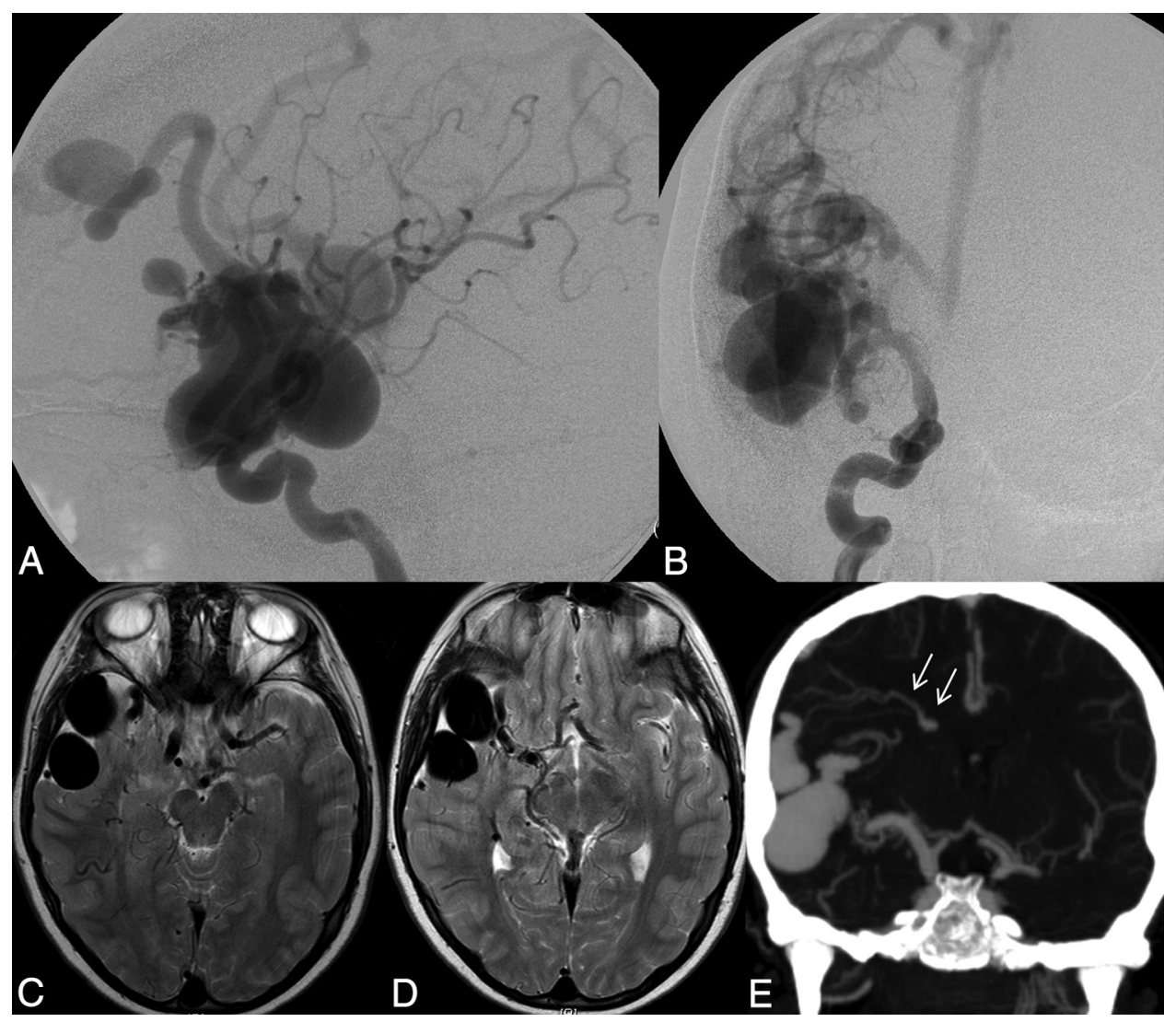

FIG 6. Right ICA catheter angiogram in lateral $(A)$ and frontal $(B)$ projections demonstrates 2 separate high-flow single-hole pial arteriovenous fistulas, 1 in the right frontal lobe and 1 in the right temporal lobe. Arterial supply to each fistula is through a single enlarged right MCA branch, which empties directly into a large venous pouch. Venous drainage is superficial, with both fistulas draining through enlarged cortical veins to the superior sagittal sinus. On axial T2-weighted MR imaging $(C$ and $D)$ venous pouches appear as flow voids and exert mass effect on the adjacent right temporal lobe. Evidence of venous congestion with venous reflux through dilated transdmedullary veins to the deep venous system is demonstrated on a coronal reconstruction of CTA (arrows).
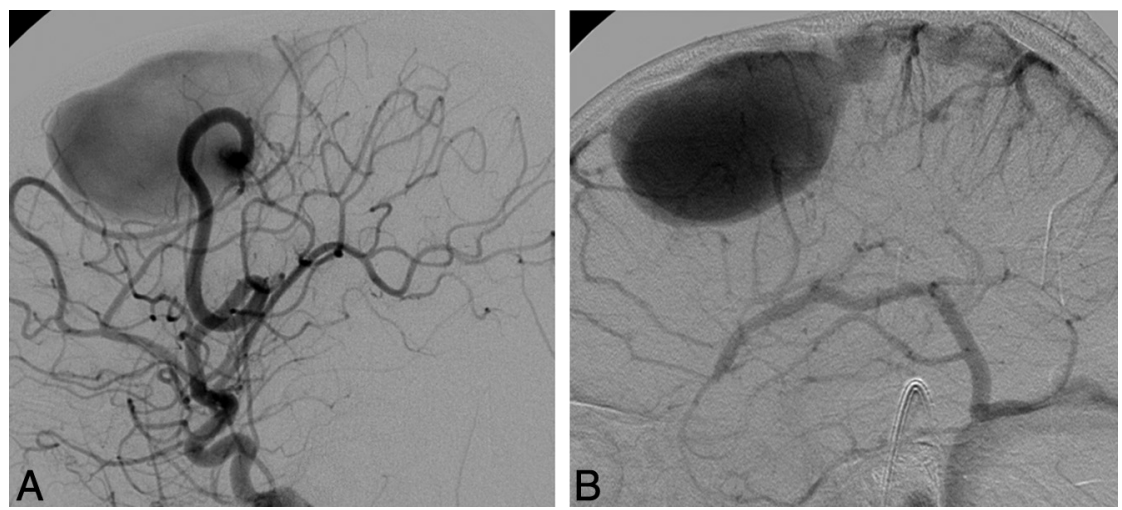

FIG 7. Lateral view of a left ICA angiogram in an 8-year-old male patient with HHT. Angiography in early arterial $(A)$ and venous $(B)$ phases demonstrates a high-flow single-hole pial arteriovenous fistula in the left frontal lobe. Arterial supply is through a single enlarged prefrontal branch of the left MCA, which empties directly into a large venous pouch. Venous drainage is superficial through an enlarged cortical vein to the superior sagittal sinus.

series that reported clinical data of the various types of brain vascular malformations in HHT were there any hemorrhages reported for patients with capillary vascular malformations. ${ }^{21}$

Whether the rate of hemorrhage of vascular malformations associated with HHT is different from that of sporadic brain
AVMs in the non-HHT population remains a matter of debate ${ }^{22}$ : In Dutch and American HHT populations, a hemorrhagic risk of $<1 \%$ was reported, ${ }^{22,23}$ a risk that was deemed significantly smaller than the presumed risk of hemorrhage in the population with sporadic AVM. However, in a retrospective study of 22,061 patient-years, similar hemorrhage rates between both groups (approximately $2.0 \%$ per patient-year) were found. ${ }^{24}$ Finally, Morgan et $\mathrm{al}^{25}$ stated that, in particular, infants and children are at high risk of sudden intracranial hemorrhage, which was further supported by the Bicêtre series, ${ }^{16}$ in which only 13 of the 50 patients had no symptoms attributable to their neurovascular manifestations. However, because these 2 latter studies are single-center studies, data may be biased due to referral base.

A potential problem of the large-scale population-based studies is that the type of vascular malformation was not further evaluated. It was assumed that all HHT-related brain vascular malformations be- 
have similarly. However, one may argue that the difference in overall bleeding rates in HHT vascular malformations compared with sporadic AVMs observed in some studies may be driven mainly by the capillary vascular malformations (ie, the most often encountered and most benign type of vascular malformation in HHT), thus biasing the results to false low rates of hemorrhage. Thus, if the presented subclassification of brain vascular malformation in HHT proves to be reflected in clinical manifestations, treatment strategies will have to be altered and individualized to the type of vascular malformation, with a more aggressive treatment in arteriovenous fistulas and/or nidus-type AVMs compared with CVMs.

HHT gene mutation information was available for $60 \%$ of our patients, and we did not observe a statistically significant association with HHT gene mutation and the type of vascular malformation or the occurrence of multiplicity. This finding fits with the clinical observation that members of the same family can present with different HHT phenotypes, though they have the same type of mutation. An important caveat of our study is that we do not have genetic information for all patients included. However, our results are also in accordance with previous reports that found brain vascular malformations to be present in all types of mutations. ${ }^{14}$

\section{CONCLUSIONS}

Our study identified several different types of brain vascular malformations in patients with HHT, which may have different natural history and treatment risks. Future studies should focus on describing the hemorrhage risks associated with these different HHT brain vascular malformations.

\section{APPENDIX}

The Brain Vascular Malformation Consortium HHT Investigator Group comprises: Murali Chakinala, Marie E. Faughnan, James R. Gossage, Katharine Henderson, Vivek Iyer, Raj Kasthuri, Helen Kim, Timo Krings, Michael T. Lawton, Doris Lin, Johannes Jurgen Mager, Justin McWilliams, Jamie McDonald, Ludmila Pawlikowska, Jeffrey Pollak, Felix Ratjen, Karen Swanson, Karel G. ter Brugge, Dilini Vethanayagam, Andrew White, Robert I. White Jr, Pearce Wilcox, and William L. Young $(\dagger)$.

$\dagger$ Deceased.

Disclosures: Helen Kim—RELATED: Grant: National Institutes of Health (U54 NS065705), ${ }^{*}$ Comments: salary support. Jeffrey Nelson—RELATED: Grant: National Institutes of Health (U54 NS065705), ${ }^{*}$ Comments: salary support. Marie E. Faughnan-RELATED: Grant: National Institutes of Health-National Institute of Neurological Disorders and Stroke National Center for Advancing Translational Sciences, Comments: detailed in the article. *Money paid to the institution.

\section{REFERENCES}

1. Bideau A, Plauchu H, Brunet G, et al. Epidemiological investigation of Rendu-Osler disease in France: its geographical distribution and prevalence. Popul 1989;44:3-22

2. Kjeldsen $\mathrm{AD}$, Vase $\mathrm{P}$, Green $\mathrm{A}$. Hereditary haemorrhagic telangiectasia: a population-based study of prevalence and mortality in Danish patients. J Intern Med 1999;245:31-39

3. Dakeishi M, Shioya T, Wada Y, et al. Genetic epidemiology of hereditary hemorrhagic telangiectasia in a local community in the northern part of Japan. Hum Mutat 2002;19:140-48

4. Fulbright RK, Chaloupka JC, Putman CM, et al. MR of hereditary hemorrhagic telangiectasia: prevalence and spectrum of cerebrovascular malformations. AJNR Am J Neuroradiol 1998;19:477-84
5. Matsubara S, Mandzia JL, ter Brugge K, et al. Angiographic and clinical characteristics of patients with cerebral arteriovenous malformations associated with hereditary hemorrhagic telangiectasia. AJNR Am J Neuroradiol 2000;21:1016-20

6. Marchuk DA, Srinivasan S, Squire TL, et al. Vascular morphogenesis: tales of two syndromes. Hum Mol Genet 2003:12(Spec No 1):R97-112

7. Guttmacher AE, Marchuk DA, White RI Jr. Hereditary hemorrhagic telangiectasia. N Engl J Med 1995;333:918-24

8. Shovlin CL, Hughes JM, Tuddenham EG, et al. A gene for hereditary haemorrhagic telangiectasia maps to chromosome 9q3. Nat Genet 1994;6:205-09

9. McAllister KA, Baldwin MA, Thukkani AK, et al. Six novel mutations in the endoglin gene in hereditary hemorrhagic telangiectasia type 1 suggest a dominant-negative effect of receptor function. $\mathrm{Hum} \mathrm{Mol}$ Genet 1995;4:1983-85

10. McAllister KA, Grogg KM, Johnson DW, et al. Endoglin, a TGF-beta binding protein of endothelial cells, is the gene for hereditary haemorrhagic telangiectasia type 1. Nat Genet 1994;8:345-51

11. Bayrak-Toydemir P, McDonald J, Markewitz B, et al. Genotype-phenotype correlation in hereditary hemorrhagic telangiectasia: mutations and manifestations. Am J Med Genet A 2006;140:463-70

12. Letteboer T, Mager J, Snijder R, et al. Genotype phenotype relationship in hereditary hemorrhagic telangiectasia. J Med Genet 2006; 43:371-77

13. Sabbà C, Pasculli G, Lenato G, et al. Hereditary hemorrhagic telangiectasia: clinical features in ENG and ALK1 mutation carriers. J Thromb Haemost 2007;5:1149-57

14. Nishida T, Faughnan M, Krings T, et al. Brain arteriovenous malformations associated with hereditary hemorrhagic telangiectasia: gene-phenotype correlations. Am J Med Genet A 2012;158A: 2829-34

15. Gallione C, Aylsworth A, Beis J, et al. Overlapping spectra of SMAD4 mutations in juvenile polyposis (JP) and JP-HHT syndrome. Am J Med Genet A 2010;152A:333-39

16. Krings T, Ozanne A, Chng SM, et al. Neurovascular phenotypes in hereditary haemorrhagic telangiectasia patients according to age: review of 50 consecutive patients aged 1 day-60 years. Neuroradiology 2005;47:711-20

17. Shovlin CL, Guttmacher AE, Buscarini E, et al. Diagnostic criteria for hereditary hemorrhagic telangiectasia (Rendu-Osler-Weber syndrome). Am J Med Genet 2000;91:66-67

18. Akers AL, Ball KL, Clancy $\mathrm{M}$, et al. Brain vascular malformation consortium: overview, progress and future directions. J Rare Disord 2013;1:5

19. Faughnan ME, Palda VA, Garcia-Tsao G, et al. International guidelines for the diagnosis and management of hereditary hemorrhagic telangiectasia. J Med Genet 2011;48:73-87

20. Bharatha A, Faughnan M, Kim H, et al. Brain arteriovenous malformation multiplicity predicts the diagnosis of hereditary hemorrhagic telangiectasia: quantitative assessment. Stroke 2012;43:72-78

21. Krings T, Ozanne A, Chng S, et al. Hereditary hemorrhagic telangiectasia. Clin Neuroradiol 2006;16:76-90

22. Maher CO, Piepgras DG, Brown RD Jr, et al. Cerebrovascular manifestations in 321 cases of hereditary hemorrhagic telangiectasia. Stroke 2001;32:877-82

23. Willemse RB, Mager JJ, Westermann CJ, et al. Bleeding risk of cerebrovascular malformations in hereditary hemorrhagic telangiectasia. J Neurosurg 2000;92:779-84

24. Easey AJ, Wallace GM, Hughes JM, et al. Should asymptomatic patients with hereditary haemorrhagic telangiectasia (HHT) be screened for cerebral vascular malformations? Data from 22,061 years of HHT patient life. J Neurol Neurosurg Psychiatry 2003;74: 743-48

25. Morgan T, McDonald J, Anderson C, et al. Intracranial hemorrhage in infants and children with hereditary hemorrhagic telangiectasia (Osler-Weber-Rendu syndrome). Pediatrics 2002;109:E12 